\title{
Genes associated with lemon floral transition and flower development during floral inductive water deficits (Abstract)
}

\author{
Jinzhi Zhang \\ College of Horticulture and Forestry Science, Huazhong Agricultural University, Wuhan, China \\ Corresponding author: Jinzhizhang@mail.hzau.edu.cn
}

\begin{abstract}
Water deficit is a key factor to induce flowering in many woody plants, but reports on the molecular mechanisms underlying floral induction by water deficit are scarce. Here, we analyzed the morphology, cytology, and different hormone levels of lemon buds during floral inductive water deficits. Higher levels of ABA were observed, and the initiation of floral bud differentiation was examined by paraffin sections analysis. A total of 1638 differentially expressed genes (DEGs) were identified by RNA sequencing. DEGs were related to flowering, hormone biosynthesis, or metabolism. The expression of some DEGs was associated with floral induction under different hormone treatments. Four genes from the phosphatidylethanolaminebinding protein family were further investigated. Ectopic expression of these genes in Arabidopsis changed the flowering time of transgenic plants. Furthermore, the $5^{\prime}$ flanking region of these genes was also isolated and sequence analysis revealed the presence of several putative cis-regulatory elements, including basic elements and hormone regulation elements. The spatial and temporal expression patterns of these promoters were investigated under water deficit treatment. Based on these findings, we propose a model for citrus flowering under water deficit conditions, which will enable us to better understand the mechanism of water deficitregulated flowering in citrus.
\end{abstract}

Keywords: Lemon floral, transition, flower development, RNA. 
